\title{
La formación del personal de las bibliotecas universitarias españolas: de la euforia a la estabilización
}

\author{
Luis Blanco Domingo*
}

Artículo recibido:

5 de diciembre de 2020

Artículo aceptado:

2 de marzo de 2021

Artículo de investigación

\section{Resumen}

El objetivo de este artículo es analizar de forma cuantitativa cómo se han implementado los cursos de formación entre el personal de las bibliotecas universitarias españolas ante el nuevo paradigma tecnológico y social al que se enfrentan. Se basa en los datos estadísticos que proporciona la Red de Bibliotecas Universitarias Españolas (REBIUN) a través de su plataforma, que permiten concluir que, salvo excepciones, no existe una clara apuesta por su dinamización, situándose la mayoría de los centros en un perfil medio o bajo, con una clara tendencia a la estabilización, y sin que la visibilidad social de la formación sea considerada como

* Universidad de Zaragoza, Facultad de Filosofía y Letras, Departamento de Ciencias de la Documentación e Historia de la Ciencia, España

Imblanco@unizar.es

INVESTIGACIÓN BIBLIOTECOLÓGICA, vol. 35, núm. 88, julio/septiembre, 2021, México, ISSN: 2448-8321 pp. 83-99 
un indicador de calidad ni de las competencias profesionales ni de los objetivos de las mismas.

Se presentan además una serie de recomendaciones para que la presencia de los cursos de formación adquiera una mayor importancia en la planificación estratégica de las bibliotecas universitarias.

Palabras clave: Formación; Personal Bibliotecario; Bibliotecas Universitarias; España

Personnel training in Spanish university libraries: from euphoria to stabilization

Luis Blanco Domingo

\section{Abstract}

This article intends to quantitatively analyze how training courses have been implemented among the staff of Spanish university libraries in the new technological and social paradigm they are facing. This is based on the statistical data provided by the Network of Spanish University Libraries (REBIUN) through its platform, which allows us to conclude that, with few exceptions, there is no clear commitment to their galvanization, being most centers in a medium or low profile, with a clear trend towards stabilization, and without the social visibility of the training being considered as an indicator of quality nor of the professional skills of them.

In addition, a series of recommendations are presented to make the presence of the training courses acquire more importance in the strategic planning of university libraries.

Keywords: Training; Library Staff; University Libraries; Spain

\section{INTRODUCCIÓN}


que supuso hace ya algunos años el impacto de internet y las nuevas tecnologías en una triple vertiente: la necesaria adaptación y conocimiento de las nuevas formas de acceso al conocimiento; la asunción de su papel como facilitadores documentales cercanos a los usuarios, y por último, la generación de automatismos que permitan solventar los problemas generados por el difícil equilibrio existente entre el acceso universal a la información y la preservación de los derechos de autor protegidos por la ley de propiedad intelectual.

La profesionalización y mejora de la capacidad técnica de los profesionales que atienden las bibliotecas universitarias se convierte por tanto en una necesidad que debe trascender el ámbito del voluntarismo para mejorar la calidad de los servicios ofertados en un entorno tan competitivo y sometido a cambios vertiginosos. La sociedad contemporánea exige al personal bibliotecario un aprendizaje continuo y constante estimulado desde las propias instituciones, que deben garantizar su solvencia y capacitación técnica. Y ello debe tener un evidente refrendo tanto en los planes estratégicos de las universidades como en los elaborados por las propias bibliotecas.

La pluralidad de fórmulas utilizadas para implementar los cursos es notoria. Se pueden canalizar a través de las convocatorias anuales de cursos para el Personal de Administración y Servicios (PAS) realizadas por los encargados de los recursos humanos, planes de formación de consorcios bibliotecarios $\mathrm{u}$ otros organismos de la propia universidad como los institutos de ciencias de la educación o los centro dedicados a las lenguas modernas o similares. Todo ello sin abandonar la idea de la autoformación específica atendiendo a las funciones y competencias del personal, bien mediante Massive Open Online Course (MOOC), cursos online o diversos tutoriales que permitan el desarrollo profesional y la mejora de la capacidad técnica de los bibliotecarios.

Este artículo pretende realizar, como objetivo general, un análisis empírico de la evolución y desarrollo de los planes de formación en las bibliotecas universitarias españolas durante el quinquenio 2015-2019, a través de los datos estadísticos que ofrece la Red de Bibliotecas Universitarias Españolas (REBIUN). Otros objetivos más específicos y concretos son conocer si existe el mismo interés en las bibliotecas universitarias dependiendo de si tienen una gestión pública o privada, la incidencia sobre el perfil profesional de los bibliotecarios, y el análisis del grado de cumplimiento de las recomendaciones de REBIUN sobre el tema entre las diferentes bibliotecas universitarias. Se vertebra a través de la formulación de varias hipótesis de trabajo a contrastar: si existen diferencias en la implementación de cursos de formación entre las bibliotecas de las universidades públicas frente a las privadas; la cuantificación de la evolución anual de los cursos y su estratificación en diversos niveles; contrastar la relación entre los cursos realizados y los impartidos en dichos centros, y por último, analizar la asistencia e interés de los mismos. 
Este tema no ha gozado de fortuna bibliográfica, siendo muy escasas las aproximaciones al mismo y mucho menos desde la óptica del análisis de datos. En el entorno anglosajón se observa una gran concienciación tanto por la necesidad de adaptar los roles y actividades del personal bibliotecario a los nuevos retos emanados de las nuevas tecnologías, como por el desarrollo personal y profesional de sus integrantes a través del desarrollo de diversos programas (Connor, 2009). En los últimos años inciden sobre todo en la vía de la formación individualizada y personalizada como medio fundamental de relación con los potenciales usuarios (Wilkinson y Lewis, 2006; Lockhart y Majal, 2012; Monroe-Gulick, Valentine y Brooks-Kiefer, 2017; Bisko et al., 2019). La propia American Library Association (ALA) estima que el aprendizaje continuo es fundamental para renovar la experiencia y las habilidades necesarias para ayudar a los usuarios en esta era de la información, y procura los recursos necesarios y las respuestas oportunas para fortalecer su desarrollo (ALA, 2020).

En España, los estudios se han dirigido bien al impacto de la dinamización y percepción de las competencias en ALFIN (Pinto, Sales y Martínez Osorio, 2009; Pinto y Uribe Tirado, 2010), o bien delimitados al ámbito de las bibliotecas universitarias catalanas y atendiendo exclusivamente a la evidencia empírica de los usuarios asistentes a cursos de formación (Martínez, 2020). Un análisis más exhaustivo lo proporcionan Gladys Matus, quien profundiza en su tesis doctoral sobre las necesidades de formación del personal bibliotecario para responder a las exigencias y necesidades de los usuarios, pero sin incorporar ningún análisis cuantitativo (Matus, 2002), y Núria Balagué, quien disecciona los proyectos y cursos implementados para el personal de la Universitat Autònoma de Barcelona (Balagué Mola y Fitó Font, 2014).

Ese mismo estudio señala algunas de las carencias que con mayor o menor intensidad todavía caracterizan a la dinamización de estos cursos: la agilidad y adaptación a unos cambios y necesidades que circulan a gran velocidad y que requieren un seguimiento constante y efectivo de su impacto; la motivación al personal para que participe de forma activa en los cursos; la dificultad de reconocer el aprendizaje informal, instando a que la curiosidad individual fomente y estimule la formación continua, y sobre todo, la inexistencia de indicadores e instrumentos capaces de evaluar la repercusión y el impacto que estos cursos tienen sobre la actividad de los profesionales (Balagué Mola y Fitó Font, 2014).

Más tangencial e indirecta es su presencia en textos sobre los recursos humanos. Son en este sentido ilustrativos los trabajos sobre las nuevas oportunidades que genera el nuevo contexto al que se enfrentan las bibliotecas 
universitarias y su impacto sobre el capital humano (Simmons-Welburn y McNeil, 2004; Crumpton, 2015, y Crumpton y Bird, 2020), o algunas aproximaciones teóricas más dirigidas a la gestión y administración de recursos (Merlo Vega, 1998; Moneda Corrochano y Peis Redondo, 1995; Torres, 1997). Por su parte, Hernández Pacheco (2020: 226 ss.) propone una visión integral de la gestión de los recursos humanos en las bibliotecas, en la que considera la formación como un paradigma basado en las exigencias del contexto y las propias organizaciones, así como en la evaluación del desempeño de cada una de las funciones y competencias del personal, la aplicación de modernas técnicas aprovechando el dinamismo de las nuevas tecnologías y en los costes que han de asumirse para llevarla a cabo.

Estima además que se dirige a cuatro niveles competenciales: "conocimientos, aplicaciones, actitudes y motivaciones" (Hernández Pacheco, 2020: 236 ss.), apostando de manera inequívoca por desarrollar un plan de formación caracterizado por programas proactivos condicionados por los objetivos y necesidades de las bibliotecas y una evaluación continua que permita su mejora constante.

Un interesante estudio sobre los perfiles profesionales de los bibliotecarios españoles, incluido el ámbito universitario, elaborado por el Consejo de Cooperación Bibliotecaria, ofrece unas conclusiones relevantes sobre la percepción que tienen sobre su formación continua. Un 58.7 \% de los centros presentan una política de formación adecuada a las necesidades de su personal, y $48.1 \%$ de las bibliotecas muestran ajustada la oferta y la demanda. En cuanto a su incidencia sobre los concursos internos de traslado, $67.10 \%$ de las bibliotecas señalan que se tienen en cuenta estos cursos, siendo un porcentaje alto pero mucho menor del que cabría esperar. Al mismo tiempo, el estudio detecta que es necesario un mayor apoyo institucional para dotar de presupuestos específicos estables a la formación continua (Tejada Artigas, 2015).

Las temáticas más solicitadas son las relacionadas con las tecnologías de la información, la normalización de estándares, la gestión de calidad, la descripción de materiales especiales en las bibliotecas, la normativa relativa a la Ley de Propiedad Intelectual, la protección de datos, los servicios de referencia a los usuarios, y la gestión cultural y difusión (Tejada Artigas, 2015).

No obstante, sí es constatable un interés institucional por la implantación de estos cursos al menos desde el punto de vista teórico. El Plan Estratégico 2020 de la Red de Bibliotecas Universitarias (REBIUN), que apuesta claramente por implementar el modelo CRAI, el cual consiste en la dinamización y conversión de las mismas en Centros de Recursos para el Aprendizaje y la Investigación en las bibliotecas universitarias españolas, señala que los bibliotecarios 
deberán aprender a ser profesionales expertos en la gestión y explotación de la información contratada, deberán apostar claramente por crear y potenciar la nueva comunicación científica y deberán gestionar la producción, edición y difusión de las publicaciones y resultados mediante políticas de acceso abierto y repositorios institucionales. La visibilidad y el impacto de la universidad y sus autores en la red es ya una necesidad detectada que las bibliotecas no pueden desaprovechar. (REBIUN, 2020)

Aunque no es muy explícito en la necesidad formativa del capital humano, no es menos cierto que concreta en una de sus misiones la promoción y estímulo de las competencias personales y profesionales de los equipos de trabajo. Su línea estratégica 2 expone la pretensión por convertir a las bibliotecas universitarias en Centros de Recursos de Aprendizaje e Investigación, la apuesta por el acceso abierto a la producción científica o implementar servicios de información y asesoramiento sobre propiedad intelectual y protección de datos para el uso de la información, lo que implica una necesaria formación del personal para afrontar tales retos, mientras que la línea 4 marca claramente la elaboración y potenciación de planes de formación y desarrollo profesional, la creación de una agenda de encuentro y sesiones de trabajo formativo y la potenciación de nuevos perfiles y competencias profesionales (REBIUN, 2020).

Estos planes, tan ambiciosos como necesarios, encuentran una repercusión no excesivamente entusiasta en las bibliotecas universitarias españolas que, en general, no dedican una especial atención a difundir ni el número ni los contenidos de los cursos realizados. No resulta muy habitual que las bibliotecas universitarias españolas ofrezcan datos exhaustivos sobre los cursos realizados, si bien este tipo de actuaciones forman parte generalmente de alguna línea de sus planes estratégicos. Algunas excepciones reseñables son la Biblioteca de la Universidad de Zaragoza, quien publica en su página web datos sobre los cursos realizados, como la duración, el número de participantes, la modalidad y el programa al que están adscritos, estableciendo una vinculación directa con los planes estratégicos y su dinamización, si bien con cierto desfase temporal (en la actualidad figuran los realizados durante el curso académico 2016-2017). O la información, menos extensa, que presenta la Biblioteca de la Universidad de Sevilla en su informe de resultados del Plan 2012-2014 (Biblioteca de la Universidad de Sevilla, 2016).

\section{Metodología}

Para la realización de este artículo hemos utilizado los datos que proporciona REBIUN, estableciendo un lapso cronológico de cinco años (2015-2019). Debe- 
mos advertir que en muchas ocasiones estos datos son parciales e incompletos, puesto que las universidades no los reportan, siendo especialmente significativa la inexistencia de los mismos en las siguientes: Alfonso X el Sabio, Camilo José Cela, Universidad Católica de Valencia, Universidad Católica San Antonio de Murcia, Universidad Complutense, Universidad Europea de Madrid, Universidad Europea Miguel de Cervantes, Universidad Francisco de Vitoria, Universitat de les Illes Balears, Universidad Internacional de la Rioja, Universidad de Jaén, Universidad de León, Universidad de Málaga, Universidad Politécnica de Cartagena, Universidad Politécnica de Madrid, Universitat Rovira i Virgili, Universidad San Pablo CEU, Universidade de Santiago de Compostela, UDIMA-Universidad a Distancia de Madrid, y Universitat de Vic.

Ello no significa que los centros reseñados no realicen cursos de mejora y perfeccionamiento de sus profesionales, sino que simplemente, y por diversas razones, no han comunicado dichos datos, lo que indudablemente puede afectar a algunas de las conclusiones de este estudio.

De la misma forma, nuestra intención es realizar un análisis exclusivamente cuantitativo, sin entrar en la profundidad, contenidos, calidad y eficacia de los cursos. Además, no hemos utilizado como variable el número de asistentes por cuanto no están segregados por cada curso realizado, sino sumados por años, por lo que no podemos cuantificar el porcentaje del personal que ha participado en los mismos sin caer en duplicidades.

También hemos utilizado como criterio de análisis la presencia de estos cursos en las páginas web de las bibliotecas universitarias. En general, se ofrece información muy descriptiva, vinculada a las distintas líneas de investigación que presentan los planes estratégicos, y a veces difícil de localizar, probablemente a que muchos de los datos figuren en la intranet de los respectivos centros.

El análisis de datos se ha efectuado utilizando el programa SPSS versión 20. Para contrastar las diferencias entre las bibliotecas universitarias, se han realizado gráficos de caja, $t$ de Student para la comparativa entre variables cualitativas y cuantitativas y correlaciones bivariadas.

Las variables creadas son:

a) Tip_uni: tipo de universidad, con dos categorías (públicas y privadas).

b) Cur_tot: número total de cursos realizados durante el quinquenio 2015-2019.

c) Imp_tot: número total de cursos impartidos por los bibliotecarios durante el quinquenio 2015-2019.

d) Cur_totr: recodificación de la variable Cur_tot en tres categorías: nivel bajo, nivel medio y nivel alto. 
e) Per_total: número total del personal bibliotecario, independientemente de su categoría, durante el quinquenio 2015-2019.

\section{Presentación y ANÁLIsis de Resultados}

\section{Diferencias entre las bibliotecas universitarias públicas y las privadas}

Tras el estudio de los datos aportados, podemos constatar como una de sus primeras conclusiones el mayor impacto que los cursos de formación tienen en las bibliotecas universitarias públicas frente a las privadas. Si observamos en primer lugar el número total de cursos realizados por las mismas durante el quinquenio 2015-2019, no hay ninguna privada entre las 10 primeras, ocupando la Universidad de Navarra el puesto undécimo (Tabla 1).

\begin{tabular}{|l|c|r|}
\hline \multicolumn{1}{|c|}{ Nombre } & Tip_uni & \multicolumn{1}{c|}{ Cur_tot } \\
\hline Universidad de Valladolid & Pública & 994 \\
\hline Universitat Autònoma de Barcelona & Pública & 918 \\
\hline UnED & Pública & 599 \\
\hline Universidad de Oviedo & Pública & 593 \\
\hline Universitat de Barcelona & Pública & 590 \\
\hline Universidad Autónoma de Madrid & Pública & 586 \\
\hline Universidad de Sevilla & Pública & 549 \\
\hline Universitat de València & Pública & 503 \\
\hline Universitat Pompeu Fabra & Pública & 462 \\
\hline Universidad Castilla-La Mancha & Pública & 395 \\
\hline
\end{tabular}

Tabla 1. Número total de cursos

Esta aseveración se ve reforzada cuando realizamos un gráfico de caja que relacione el tipo de biblioteca con el total de cursos (Figura 1). Gracias al mismo colegimos que las bibliotecas de las universidades públicas presentan una media de cursos de formación más alta que las privadas, si bien ambas concentran sus acciones pedagógicas en la horquilla más baja (entre 170-220 en las públicas y 80-100 en las privadas). Hay dos valores extremos en las universidades públicas, que representan a las bibliotecas universitarias de Valladolid (994) y la Autònoma de Barcelona (918), cuyo número sobrepasa con creces las medias del resto, que presentan una mayor cercanía y estabilidad. 


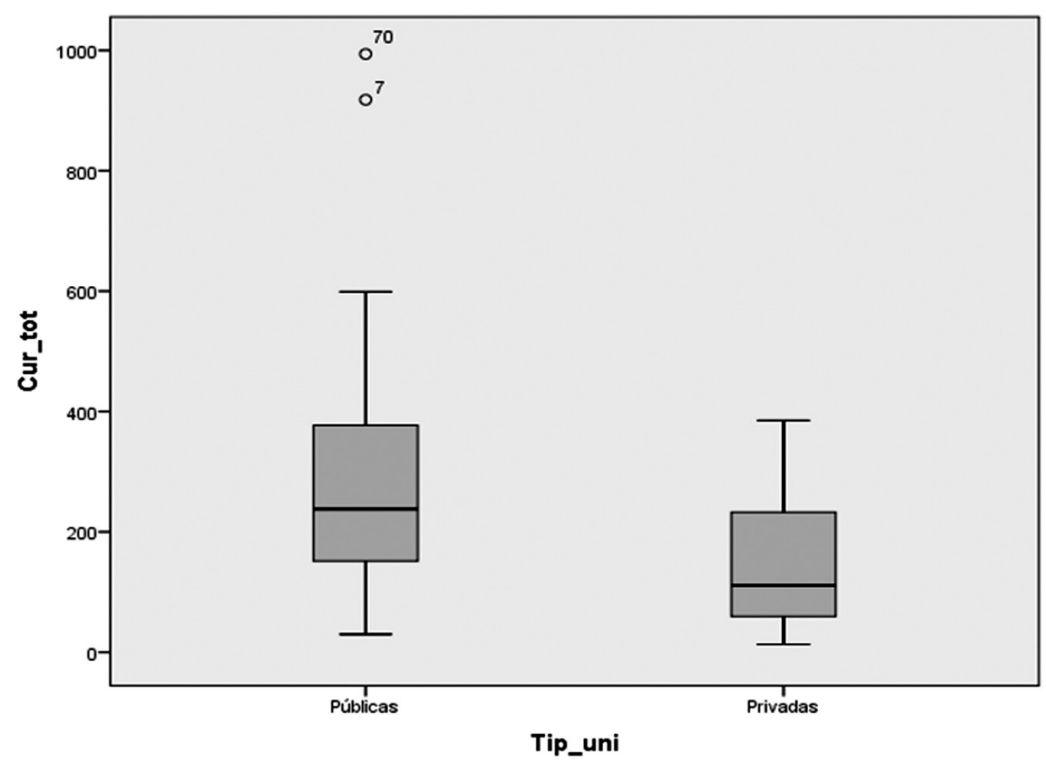

Figura 1. Gráfico de cajas tipo de biblioteca y número total de cursos

Para reafirmar nuestras conclusiones, hemos realizado una $t$ de Student que contrasta las medias de los cursos realizados por las dos categorías de bibliotecas universitarias (Tabla 2).

\begin{tabular}{|l|c|c|c|r|r|}
\hline \multicolumn{7}{|c|}{ Estadísticos de grupo } \\
\hline \multirow{2}{*}{ Cur_tot } & Tip_uni & N & Media & Desviación típ. & Error típ. de la media \\
\cline { 2 - 6 } & Públicas & 40 & 302,73 & 219,284 & 34,672 \\
\cline { 2 - 6 } & Privadas & 15 & 153,20 & 111,412 & 28,767 \\
\hline
\end{tabular}

\begin{tabular}{|c|c|c|c|c|c|c|c|c|c|c|}
\hline \multicolumn{11}{|c|}{ Prueba de muestras independientes } \\
\hline & & \multicolumn{2}{|c|}{$\begin{array}{l}\text { Prueba de Levene para la } \\
\text { igualdad de varianzas }\end{array}$} & \multicolumn{7}{|c|}{ Prueba T para la igualdad de medias } \\
\hline & & \multirow[b]{2}{*}{$\mathbf{F}$} & \multirow[b]{2}{*}{ sig. } & \multirow[b]{2}{*}{$t$} & \multirow[b]{2}{*}{ و ا } & \multirow[b]{2}{*}{ Sig. (bilateral) } & \multirow{2}{*}{$\begin{array}{l}\text { Olferencla de } \\
\text { medias }\end{array}$} & \multirow{2}{*}{$\begin{array}{l}\text { Error típ. de la } \\
\text { diferencia }\end{array}$} & \multicolumn{2}{|c|}{$\begin{array}{c}\text { 95\% Intervalo de confianza para } \\
\text { la diferencia }\end{array}$} \\
\hline & & & & & & & & & Inferior & Superior \\
\hline \multirow[t]{2}{*}{ Cur_tot } & $\begin{array}{l}\text { Se han asumido } \\
\text { varianzas iguales }\end{array}$ & 3.247 & .077 & 2,512 & 53 & .015 & 149,525 & 59,532 & 30,119 & 268,931 \\
\hline & $\begin{array}{l}\text { No se han asumido } \\
\text { varianzas iguales }\end{array}$ & & & 3,319 & 47,919 & .002 & 149,525 & 45,052 & 58,939 & 240,111 \\
\hline
\end{tabular}

Tabla 2. $t$ de Student de los cursos realizados 
Gracias a esta prueba detectamos que, asumiendo la homogeneidad de varianzas gracias a Levene $(p=0.077)$, existen diferencias estadísticamente significativas entre las medias de las universidades públicas y privadas ( $p=0.015$ ), siendo la media de las primeras de 302.73 frente a 153.20 en las privadas. Podemos concluir además que, con un nivel de confianza de $95 \%$, las bibliotecas universitarias públicas realizan entre 30 y 269 cursos más que las privadas.

Sin embargo, si analizamos las medias de los cursos impartidos por el personal bibliotecario (Tabla 3), observamos que, asumiendo la homogeneidad de varianzas gracias a Levene $(p=0.131)$, no existen diferencias estadísticamente significativas entre las bibliotecas universitarias públicas y las privadas, puesto que el valor de $p$ es superior a $0.05(p=0.116)$.

\begin{tabular}{|r|c|c|c|r|r|}
\hline \multicolumn{7}{|c|}{ Estadísticos de grupo } \\
\hline \multirow{2}{*}{ Imp_tot } & Tip_uni & N & Media & Desviación típ. & \multicolumn{1}{c|}{ Error típ. de la media } \\
\cline { 2 - 6 } & Públicas & 36 & 57,14 & 71,212 & 11,869 \\
\cline { 2 - 6 } & Privadas & 14 & 24,64 & 41,085 & 10,980 \\
\hline
\end{tabular}

\begin{tabular}{|c|c|c|c|c|c|c|c|c|c|c|}
\hline \multicolumn{11}{|c|}{ Prueba de muestras independlentes } \\
\hline & & \multicolumn{2}{|c|}{$\begin{array}{l}\text { Prueba de Levene para la } \\
\text { igualdad de varianzas }\end{array}$} & \multicolumn{7}{|c|}{ Prueba T para la igualdad de medias } \\
\hline & & \multirow[b]{2}{*}{$\mathbf{F}$} & \multirow[b]{2}{*}{ Sig. } & \multirow[b]{2}{*}{$t$} & \multirow[b]{2}{*}{ gl } & \multirow[b]{2}{*}{ Sig. (bilateral) } & \multirow{2}{*}{$\begin{array}{l}\text { Diferencia de } \\
\text { medias }\end{array}$} & \multirow{2}{*}{$\begin{array}{l}\text { Error típ. de la } \\
\text { diferencia }\end{array}$} & \multicolumn{2}{|c|}{$\begin{array}{c}95 \% \text { Intervalo de confianza para } \\
\text { la diferencia }\end{array}$} \\
\hline & & & & & & & & & Inferior & Superior \\
\hline Imp_tot & $\begin{array}{l}\text { Se han asumido } \\
\text { varianzas iguales }\end{array}$ & 2,362 &, 131 & 1,601 & 48 &, 116 & $\overline{32,496}$ & 20,302 & $-8,325$ & 73,317 \\
\hline & $\begin{array}{l}\text { No se han asumido } \\
\text { varianzas iguales }\end{array}$ & & & 2,010 & 40,559 & ,051 & 32,496 & 16,169 &,- 168 & 65,161 \\
\hline
\end{tabular}

Tabla 3. $t$ de Student de cursos impartidos por el personal bibliotecario

\section{Evolución y medias de los cursos}

El segundo aspecto que hemos considerado oportuno incluir en nuestro estudio se dirige a cuantificar tanto la evolución anual de los cursos como su gradación. Para el primero de los indicadores, hemos elegido las 10 primeras universidades como representación cualificada de las restantes, realizando un gráfico que incorpora el número anualizado (Figura 2). Se observa en primer lugar cierta estabilidad, puesto que todos se sitúan en una horquilla entre 50 y 250, si bien tanto la biblioteca de la Universidad de Valladolid (pasa de 165 en 2018 a 269 en 2019) como la de la Autònoma de Barcelona (de 196 en 2018 a 255 en 2019) experimentan unas notables subidas en 2019, frente a descensos tan significativos como el que ofrecen la Universitat de Barcelona (de 195 en 2018 a 125 en 2019) o la UNED (de 133 en 2018 a 87 en 2019). 


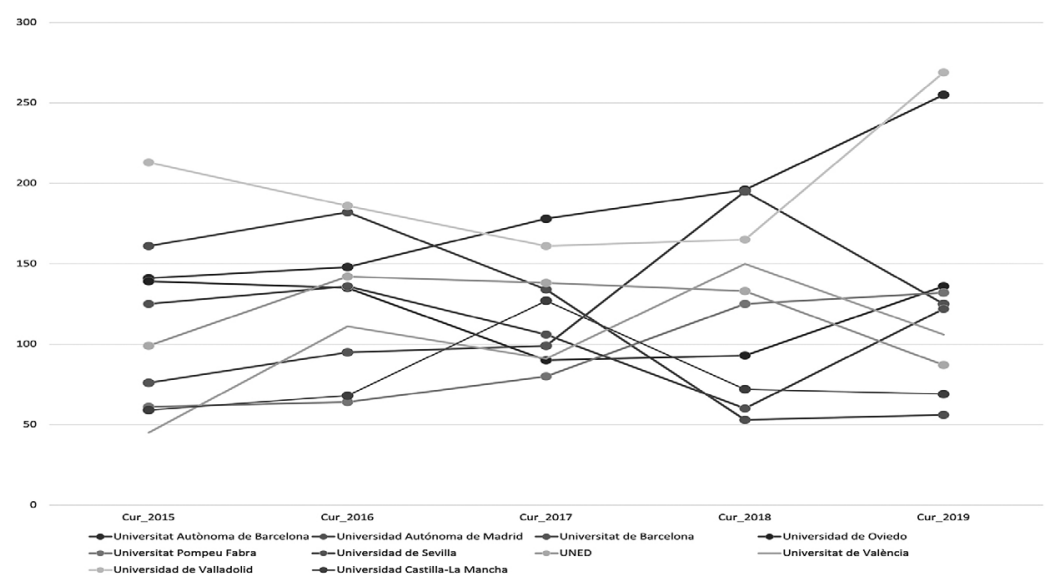

Figura 2. Evolución cursos 2015-2019

En lo que se refiere a la gradación de cursos, hemos establecido tres niveles: de 0 a 200 bajo, de 201 a 400 medio y a partir de 401 alto. Una distribución de frecuencias posibilita concluir que son muy escasas las bibliotecas universitarias que durante el quinquenio 2015-2019 han realizado más de 400 cursos (16.4\%), frente a las que sitúan su horquilla en el nivel más bajo (45.5\%) o medio (38.2\%) (Tabla 4). Ello constata que, pese al teórico interés suscitado por desarrollar cursos, idea que encuentra acomodo en los planes estratégicos de los centros, no hay una traslación práctica de esas intenciones, o al menos con la intensidad que se precisa.

Cur_totr

\begin{tabular}{|ll|r|r|r|r|}
\hline & Frecuencia & Porcentaje & $\begin{array}{c}\text { Porcentaje } \\
\text { válido }\end{array}$ & $\begin{array}{c}\text { Porcentaje } \\
\text { acumulado }\end{array}$ \\
\hline Válidos & Bajo & 25 & 32,9 & 45,5 & 45,5 \\
& Medio & 21 & 27,6 & 38,2 & 83,6 \\
& Alto & 9 & 11,8 & 16,4 & 100,0 \\
& Total & 55 & 72,4 & 100,0 & \\
Perdidos & Sistema & 21 & 27,6 & & \\
Total & & 76 & 100,0 & & \\
\hline
\end{tabular}

Tabla 4. Distribución de frecuencias gradación de cursos 


\section{Relación entre los cursos realizados e impartidos en las bibliotecas universitarias}

Para comprobar si existe alguna relación entre los cursos realizados en las bibliotecas universitarias y los impartidos por el personal de las mismas, hemos utilizado una correlación bilateral entre las dos variables cuantitativas (Tabla 5), cuyo resultado es que hay diferencias estadísticamente significativas ( $p=0.000$ ), existiendo una vinculación entre ellas de prácticamente $74 \%$.

\begin{tabular}{|c|c|c|c|}
\hline \multicolumn{4}{|c|}{ Correlaciones } \\
\hline & & Cur_tot & Imp_tot \\
\hline Cur_tot & $\begin{array}{l}\text { Correlación de Pearson } \\
\text { Sig. (bilateral) } \\
\text { N }\end{array}$ & $\begin{array}{r}1 \\
55\end{array}$ & $\begin{array}{r}, 737 \\
, 000 \\
49\end{array}$ \\
\hline Imp_tot & $\begin{array}{l}\text { Correlación de Pearson } \\
\text { Sig. (bilateral) } \\
\text { N }\end{array}$ & $\begin{array}{r}, 737^{\mathrm{m}} \\
, 000 \\
49\end{array}$ & $\begin{array}{r}1 \\
50\end{array}$ \\
\hline
\end{tabular}

Tabla 5. Correlación bivariada entre cursos realizados e impartidos

\section{Asistencia del personal bibliotecario}

Debido a las características propias de los datos suministrados por las bibliotecas universitarias a REBIUN, no podemos obtener la comparativa entre el número de personal bibliotecario que asiste a los cursos y el número total de los mismos, puesto que al realizar la suma global, no los singulariza, dándose el caso de que los mismos individuos pueden aparecer varias veces.

Sin embargo, sí es posible diferenciar qué bibliotecas universitarias tienen una mayor asistencia y vincularla con el número de cursos realizados. En general, aparecen de nuevo las que más cursos imparten, aunque en orden cambiado, y no siempre vinculado a un mayor número de personal. Entre las 10 primeras, la que dispone de más personal es la biblioteca de la Universidad de Sevilla (1 131); sin embargo, se ve rebasada por la Biblioteca de la Universidad Autònoma, con menos efectivos (850). Del mismo modo, la Universitat de Barcelona, pese a disponer de 1109 personas, ocupa la cuarta plaza. Y a la inversa, la biblioteca de la Universidad Pública de Navarra, contando de un menor número de personal que otras (305), se sitúa en una significativa séptima plaza. 
En este apartado sí encontramos una biblioteca universitaria privada entre las 10 primeras, concretamente la Universidad de Navarra, pese a que su personal no se encuentra entre los más numerosos (dispone de una plantilla de 321 efectivos.)

Si tratamos de averiguar la existencia de una relación entre el número de cursos totales y el número de personal de las bibliotecas universitarias, observamos que existen diferencias estadísticamente significativas $(p=0.000)$, y gracias a la correlación de Pearson, estimamos que ambas variables no son independientes, siendo su vinculación de $60 \%$.

\section{Correlaciones}

\begin{tabular}{|rl|r|r|}
\hline & & Per_tot & Cur_tot \\
\hline Per_tot & Correlación de Pearson & 1 &, $600^{\prime \prime}$ \\
& Sig. (bilateral) & &, 000 \\
& N & 63 & 55 \\
\hline Cur_tot & Correlación de Pearson &, $600^{\mathrm{N}}$ & 1 \\
& Sig. (bilateral) &, 000 & \\
& $\mathrm{~N}$ & 55 & 55 \\
\hline
\end{tabular}

**. La correlación es significativa al nivel 0,01 (bilateral).

Tabla 6. Correlación de Pearson

\section{Discusión}

Pese al evidente interés que suscita el tema para REBIUN, plasmado en sus planes estratégicos en vigor, se evidencia, a través del análisis expuesto, que su dinamización ha sufrido una evidente tendencia a la estabilización en su número e intensidad, quizá por la conjunción de algunas de las tendencias enunciadas en el apartado "La formación del personal".

Tampoco resulta frecuente en la literatura científica ofrecer esta vertiente cuantitativa del proceso, capaz de objetivar sus resultados, y la adecuación a un contexto condicionado por la implosión de las nuevas tecnologías. Los textos dedicados al estudio de los recursos humanos en las bibliotecas universitarias aluden de forma tangencial y difuminada a estos hechos, más de una manera teórica que práctica.

Todo ello pese a la evidencia de que el perfil profesional de los bibliotecarios españoles requiere, para su consolidación y promoción, de una apelación continua a la formación como elemento indispensable, situación a veces no debidamente comprendida por las instituciones en las que desarrollan su labor, pese a las recomendaciones de REBIUN en ese sentido. 
El análisis cuantitativo de los cursos realizados por las bibliotecas universitarias españolas durante el quinquenio 2015-2019 genera cierta perplejidad, puesto que a pesar de los intentos de dinamización realizados desde el plan estratégico de REBIUN, y la decidida apuesta por su implementación a nivel teórico desde las diferentes bibliotecas universitarias, no parece existir un interés generalizado para su traslación a la práctica. Los retos del nuevo paradigma generado por la explosión de las nuevas tecnologías e internet, los usos y consumos de los usuarios, o la aparición de múltiples cauces de visibilidad de la documentación científica precisan de una respuesta profesional de los bibliotecarios, cuya función y competencias ha experimentado una notable evolución que exige su traslación formativa.

Y sin embargo, no sólo se observa una estabilización a la baja del número de cursos, sino que es constatable su escasa presencia en la visualización social de las actividades de las bibliotecas universitarias. Lejos de convertirse en un indicador de calidad, los escasos centros que ofrecen información lo hacen de forma parcial y complicada de localizar en las páginas web. E incluso el llamativo silencio de algunas de ellas en la comunicación de los datos a REBIUN es una muestra significativa de ese distanciamiento.

Un amplio porcentaje de bibliotecas universitarias (concretamente $45.5 \%$ ) realizaron un número de cursos que hemos considerado de nivel bajo, con una horquilla situada entre 0 y 200, siendo únicamente $16.4 \%$ aquellas que sitúan sus umbrales entre 401 y 800 . Aunque la cantidad no denota calidad, al menos muestra la predisposición e interés de los centros por dinamizar la formación continua de su personal, siendo en este sentido decepcionante.

Si nos atenemos exclusivamente a la cuantificación de los cursos, en primer lugar, llama la atención la enorme desigualdad y heterogeneidad que presentan. Las bibliotecas de la Universidad de Valladolid y la Autònoma de Barcelona destacan sobremanera sobre el resto, que mantiene cierta tendencia al descenso.

En segundo lugar, resultan claras y notables las diferencias entre la preocupación e interés de las bibliotecas universitarias públicas frente a las privadas, menos atraídas por su aplicación y desarrollo, si bien esta diferenciación no se constata cuando nos referimos a los cursos impartidos por el personal de las mismas.

En tercer lugar, la mayor parte de las bibliotecas universitarias sitúan el número de cursos entre 0 y 200 (45.5\%), o entre 201 y 400 (38.2\%), siendo claramente minoritarias aquellas que superan los 400 (tan sólo $16.4 \%$ ).

En cuanto al número de personas que participan en los cursos de formación, y tomando en consideración el señalado hándicap que representa 
la imposibilidad de cuantificar de forma individual el número de asistentes a cada uno de ellos, podemos concluir que es evidente la relación entre el número de cursos y la cantidad de personal integrado en las plantillas de las bibliotecas universitarias, de tal forma que la dimensión de los centros condiciona las cifras de cursos realizados.

Todo ello nos estimula para establecer una serie de recomendaciones destinadas a mejorar la incidencia y repercusión de los cursos de formación. En primer lugar, es importante intentar persuadir a las bibliotecas universitarias españolas de la necesidad de considerar la formación continua de su personal como un elemento fundamental tanto para la mejora del clima laboral como por la trascendencia e impacto en la mejora de la calidad de sus prestaciones y servicios, lo que se traducirá en un mayor respaldo social y un crecimiento en la satisfacción tanto de sus usuarios como de la sociedad a la que sirven.

En segundo lugar, los programas activos de formación deben tener una presencia relevante en la planificación estratégica de las bibliotecas universitarias, en consonancia con los objetivos que señale el centro educativo superior al que pertenecen y sirven. La realidad líquida en la que nos desenvolvemos exige una proactividad en la actuación de los profesionales, y abarcar las múltiples áreas de intervención en las que la presencia de los bibliotecarios puede ser efectiva y eficaz.

En tercer lugar, los gestores de las bibliotecas universitarias deben aprovechar las sinergias de las encuestas de clima laboral elaborados en sus centros u otro tipo de herramientas o indicadores para detectar cuáles son las necesidades reales de los profesionales, y trasladarlas a programas capaces de satisfacer esas carencias. Todo ello sin olvidar la enorme importancia del contexto tecnológico y social en que se desenvuelven.

En cuarto lugar, reafirmar y reformar la convivencia de las bibliotecas con los órganos principales de las universidades (rectores, consejo social), que se han de convertir en aliados y sustento de la planificación generada tanto desde el punto de vista económico como del respaldo social e institucional.

Su importancia no sólo se reduce a la mejora y promoción del desarrollo profesional de los bibliotecarios, ni tampoco únicamente a la adaptación a las nuevas funciones y competencias que han de asumir como resultado de la incidencia de un nuevo contexto tecnológico marcado por la gestión de la información, sino también como fórmula que garantice la supervivencia de las bibliotecas universitarias en un entorno abiertamente competitivo, en el que el dominio del proceso técnico y de las funciones tradicionalmente atribuidas a los bibliotecarios no resultan suficientes y paulatinamente se tornan más prescindibles. 


\section{REFERENCIAS}

ALA (American Library Association). 2020. Staff development. Tools, publications $\mathcal{E}$ resources. http://www.ala.org/tools/atoz/continuing-education

Balagué Mola, Núria y Neus Fitó Font. 2014. "Actualización profesional en el entorno universitario: la formación en el Servicio de Bibliotecas de la Universidad Autónoma de Barcelona”. BiD, textos universitaris de biblioteconomia i documentació 32 (junio). http://bid.ub.edu/pdf/32/es/nbalague2.pdf

Biblioteca de la Universidad de Sevilla. 2016. Plan Estratégico de la BUS 2012-2014 (ext. 2015). Informe de resultados. https://bib.us.es/sites/bib3.us.es/files/Biblioteca_Universitaria/informe_resultados_pe2012_2015.pdf

Biblioteca de la Universidad de Zaragoza. 2017. Formación del personal BUZ. https://biblioteca.unizar.es/conocenos/formacion-del-personal-buz

Bisko, Lynne, Heather Buchansky, Brian C. Gray y E. Gail Reese. 2019. Personal librarians: building relationships for student success. Santa Barbara: Libraries Unlimited.

Connor, Elizabeth, ed. 2009. An introduction to staff development in academic libraries. New York: Routledge.

Crumpton, Michael A. 2015. Strategic Human Resource Planning for Academic Libraries: Information, Technology and Organization. Waltham: Chandos Publishing.

Crumpton, Michael A. y Nora J. Bird, eds. 2020. Emerging Human Resource Trends in Academic Libraries. Londres: Rowman \& Littlefield.

Hernández Pacheco, F. 2020. Dirección de recursos bumanos en bibliotecas y otras instituciones. México: Universidad Nacional Autónoma de México, Instituto de Investigaciones Bibliotecológicas y de la Información. http://ru.iibi.unam.mx/jspui/bitstream/IIBI_UNAM/L222/1/L227.pdf

Lockhart, Janine y Sulaiman Majal. 2012. "The effect of library staff training and development on the user experience: a case study at the Cape Peninsula University of Technology (CPUT)". Proceedings of the IATUL Conferences. Paper 44.

Martínez, Dídac. 2020. "Les biblioteques universitàries a Catalunya (2018-2019): actuacions i reptes de futur". Anuario de Bibliotheques, llibres i Lectura, 65-88.

Matus, Gladys. 2002. "Los recursos humanos de las bibliotecas universitarias: exigencias, conflictos y necesidades de información". Tesis doctoral. Universitat de Lleida. Facultat de Ciències de l'Educaciò, España.

Merlo Vega, José Antonio. 1998. "Fundamentos de gestión de bibliotecas universitarias". Boletin ANABAD 48 (2): 261288.

Moneda Corrochano, Mercedes de la, y Eduardo Peis Redondo. 1995. "Bibliotecas universitarias: recursos humanos y formación y el mercado de trabajo”. Boletín de la Asociación Andaluza de Bibliotecarios 41, 4970.

Monroe-Gulick, Amalia, Greta Valentine y Jamene Brooks-Kiefer. 2017. "You need to have a Street beat: a qualitative study of faculty research needs and challenges". Portal: Libraries and the Academy 17 (4): 777-802 
Pinto, María, Dora Sales y Pilar Martínez Osorio. 2009. “El personal de la biblioteca universitaria y la alfabetización informacional: de la autopercepción a las realidades y retos formativos". Revista Española de Documentación Científica 21 (1): 6080.

Pinto, María, y Alejandro Uribe Tirado. 2010. "Formarnos y autoformarnos en alfabetización informacional. Un programa de mentorización en bibliotecas universitarias-CRAI". Investigación Bibliotecológica 24 (52): 63-95. http://doi.org/10.22201/iibi.0187358xp.2010.52.27453

REBIUN (Red de Bibliotecas Universitarias Españolas). 2020. III Plan Estratégico de REBIUN 2020.

https:/www.REBIUN.org/sites/default/files/2017-11/Plan\%20Estrat\%C3\%A9gico\%20REBIUN.pdf

Simmons-Welburn, Janice y Beth McNeil, eds. 2004. Human resource management in today's academic library: meeting challenges and creating opportunities. Westport, Conn.: Libraries Unlimited.

Tejada Artigas, C. M., coord. 2015. Formación continua en el Sistema Español de Bibliotecas: Resultados de una encuesta. Madrid: Ministerio de Educación, Cultura y Deporte.

https://digital.csic.es/bitstream/10261/130227/1/Formacion_continua_SEB_ CCB.pdf

Torres, Marta, coord. 1997. Los recursos bumanos en las bibliotecas universitarias. Madrid: REBIUN.

http://REBIUN.xercode.es/xmlui/bitstream/handle/20.500.11967/57/los\%20rr\%20 humanos $\% 20$ en $\% 20$ las $\% 20$ bus $\% 20 \% 281 \% 29$.pdf? sequence $=1 \&$ isAllowed=y

Wilkinson, Frances C. y Linda K. Lewis. 2006. "Training programs in academic libraries: Continuous learning in the information age". College \& Research Libraries News 67 (6): 356 - 358, 365.

Para citar este texto:

Blanco Domingo, Luis. 2021. "La formación del personal de las bibliotecas universitarias españolas: de la euforia a la estabilización”. Investigación Bibliotecológica: archivonomía, bibliotecología e información 35 (88): 83-99.

http://dx.doi.org/10.22201/iibi.24488321xe.2021.88.58383 